\title{
TRADICIONES TECNOLÓGICAS DEL PERÍODO ALFARERO TEMPRANO DE CHILE CENTRAL. UN ESTUDIO DE BORDES, MATERIAS PRIMAS Y PASTAS DE VASIJAS DE COCINA EN LA MICRORREGIÓN DE ANGOSTURA
}

\author{
TECHNOLOGICAL TRADITIONS FROM THE EARLY CERAMIC PERIOD \\ IN CENTRAL CHILE. RIM, RAW MATERIAL AND FABRIC ANALYSES \\ OF COOKING VESSELS IN THE ANGOSTURA MICRO REGION
}

\author{
Fernanda Falabella ${ }^{1}$, Lorena Sanhueza ${ }^{1}$, Itací Correa ${ }^{2}$,Eugenia Fonseca ${ }^{3}$, \\ Cody C. Roush ${ }^{4}$ y Michael D. Glascock ${ }^{4}$
}

\begin{abstract}
En sociedades de baja escala, como los grupos del período Alfarero Temprano de Chile Central, el registro cerámico es un buen referente de las tradiciones reproducidas al interior de comunidades de alfareras(os) y de la circulación de sus saberes. Este trabajo evalúa a qué escala espacial se compartían esos saberes y su relación con los componentes culturales Bato y Llolleo, para entender las unidades sociales mínimas y su articulación en niveles sociales mayores. Basados en el análisis de rasgos tecnológicos evidenciados en fragmentos de vasijas de cocina, como morfología de bordes, gestos técnicos de manufactura, composición de pastas y procedencia de materias primas, se observan patrones diferenciados a nivel del grupo cultural, de las comunidades locales y de los conjuntos residenciales.
\end{abstract}

Palabras claves: tecnología cerámica, activación neutrónica, Chile Central, Bato, Llolleo.

Social organization in small scale societies, such as Early Ceramic Period groups in Central Chile, can be understood from a bottom up perspective, exploring information in their smallest social units. In these societies ceramic data are good approximations to technological traditions reproduced in pottery communities. In this paper we explore whether ceramic traditions were shared beyond the residential compounds and defined cultural groups (Bato and Llolleo). We analyzed rim and paste attributes in cooking vessels looking for technical gestures, paste recipes and raw material provenience. The results show patterns at the local community level for raw material procurement, patterns according to cultural groups for paste recipes and some technical gestures shared at the co-residential level.

Key words: Ceramic technology, neutron activation, Central Chile, Bato, Llolleo.

Los complejos culturales Bato y Llolleo se desarrollaron durante el período Alfarero Temprano (200 a 1.000/1.200 d.C.) en el centro de Chile, desde zonas cercanas al río Aconcagua por el norte hasta al menos la cuenca de Rancagua por el sur, con asentamientos distribuidos a lo largo de la costa, los valles de la cordillera de la Costa y de los Andes y el valle Central (Figura 1) (Falabella y Stehberg 1989; Sanhueza et al. 2003). Estas unidades arqueológicas son el referente espacial y material de dos grupos prehispánicos de Chile Central que, si bien presentan varias diferencias culturales, se inscriben dentro de lo que se ha conocido en antropología como "sociedades simples" o "de baja escala", sin jerarquías institucionalizadas, un patrón de asentamiento disperso y con prácticas hortícolas. La organización social de estos grupos se configura sobre la base de distintos niveles de cohesión. El más amplio y laxo es el que involucra las redes de relaciones a nivel de la región y que

1 Departamento de Antropología, FACSO, Universidad de Chile, Ignacio Carrera Pinto 1045, Nuñoa, Santiago, Chile. ffalabella@vtr.net; loresan@uchile.cl

2 Programa de Doctorado en Arqueología, Facultad de Filosofía y Letras, Universidad de Buenos Aires, Buenos Aires, Argentina. kusvetiver@gmail.com

3 Laboratorio Servicio Nacional de Geología y Minería, Santiago, Chile. efonseca@sernageomin.cl

4 University of Missouri-Research Reactor Center, Columbia, Missouri, EE.UU. RoushC@missouri.edu; glascockm@missouri.edu 


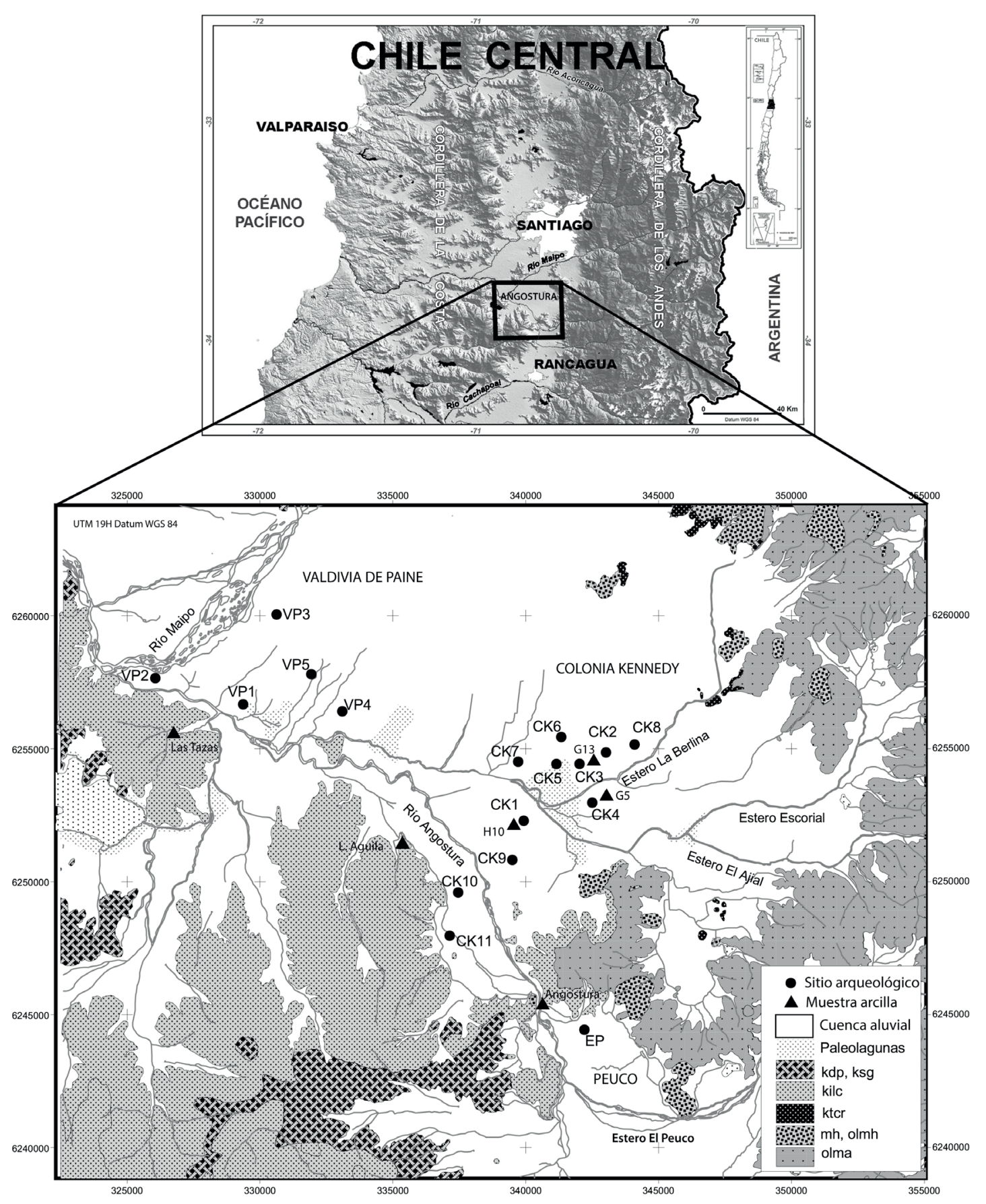

Figura 1. Mapa de la microrregión de Angostura con las formaciones geológicas principales (según Sellés y Gana 2001), sitios arqueológicos y lugar de obtención de muestras de depósitos arcillosos.

Map of the Angostura micro region showing the main geological formations (after Sellés and Gana 2001), archaeological sites, and the location of clay sources. 
dan la identidad al complejo cultural (Falabella y Stehberg 1989; Falabella y Sanhueza 2005-2006; Planella y Falabella 1987). Formas de cohesión intermedias estarían representadas por conjuntos de asentamientos a lo largo de la costa, de un curso de agua, o de un valle, en el caso Llolleo (Sanhueza 2004; Sanhueza y Falabella 2007; Sanhueza et al. 2007); o bien por quienes ocupan ciertos espacios cercanos, diferenciándose paulatinamente de norte a sur, como en el caso Bato (Sanhueza 2013). Los niveles de mayor cohesión, que debieran corresponder a unidades corresidenciales y comunidades locales, han sido poco estudiadas, no obstante sean estas instancias los referentes esenciales para el desarrollo de las actividades diarias, la cooperación económica, inversión en tecnología, disminución de riesgos, y un nivel significativo de integración social (Johnson y Earle 1987).

Con miras a comprender este tipo de relaciones más cercanas, en particular la integración social a nivel de unidades corresidenciales, de la localidad y de la microrregión, se realizó un estudio espacial sobre la distribución de los asentamientos en la zona de Angostura, en el extremo meridional de la cuenca de Santiago (Falabella et al. 2012) (Figura 1). Los resultados dejaron en evidencia que la distribución de los asentamientos domésticos no fue al azar; existían agrupamientos que conformaban conjuntos corresidenciales y otros a nivel de la localidad (Cornejo et al. 2012; Falabella, Cornejo, Correa y Sanhueza 2014). Estos agrupamientos por sí solos, no obstante, no eran suficientes para desprender de ellos, la existencia de redes de relaciones o proximidad social. Para ir un paso más allá en la interpretación, consideramos necesario evaluar si la proximidad o distancia física entre las agrupaciones de conjuntos residenciales encuentra un correlato en algún tipo de semejanza o distancia entre tradiciones tecnológicas entre ellos o si se advierten relaciones sociales mediante evidencias de circulación de objetos o materias primas.

Este trabajo se enfocó a la alfarería doméstica de los sitios de la microrregión de Angostura, con el fin de evaluar a qué escala espacial se compartían las tradiciones tecnológicas alfareras y su relación con los componentes culturales Bato y Llolleo. Específicamente, intentaremos responder (a) si existen tradiciones tecnológicas diferenciadas en la muestra, (b) cómo se distribuyen en el espacio y (c) si comparten recursos y fuentes de materias primas. Con ello precisaremos si las evidencias cerámicas apoyan la existencia de comunidades de prácticas alfareras a nivel del conjunto residencial, de la comunidad local, de los grupos culturales, de la localidad, y/o de la microrregión.

\section{Premisas y Expectativas}

Partimos de dos supuestos fundamentales de la antropología de la tecnología: que la vasija es un producto social, resultado de las opciones que toman los alfareros a lo largo de la cadena operativa (Lemonnier 1992) y que los particulares modos de hacer se expresan en estilos tecnológicos que devienen en el proceso de manufactura permeando todos los atributos del artefacto (Lechtmann 1977; Lemonnier 1986). Estos modos de hacer se internalizan durante el aprendizaje en las comunidades de alfareros y se reproducen en la práctica del oficio a lo largo del tiempo, generando tradiciones tecnológicas. Como consecuencia, es posible suponer que los artefactos y sus atributos manifiesten regularidades o patrones relacionados con estas tradiciones, vinculados a distintos factores. De una manera bastante simplificada esperaríamos encontrar patrones derivados de (a) decisiones sobre cómo debe ser la apariencia de cada categoría de vasija, (b) decisiones relacionadas con la cualidad de las materias primas y acciones en el proceso de formatización y (c) del uso que se pretende dar a la vasija. Por cierto, también esperamos que existan patrones relacionados con la disponibilidad y distribución de las fuentes de materia prima en el entorno natural. Los primeros suelen manifestarse en atributos visibles como formas y decoraciones, siendo diferentes tanto entre los componentes Bato y Llolleo en Chile Central a nivel regional como dentro de cada complejo cultural a nivel microrregional (Sanhueza 2004; Sanhueza y Falabella 2007). Los segundos expresan los modos de hacer particulares y la gestualidad corporal que se adquiere durante el aprendizaje en el seno de las comunidades de prácticas (Lave y Wenger 1991). Este tipo de gestos, plasmados en atributos ocultos, son los que mejor develan las unidades mínimas de organización de la producción alfarera (Gosselain 2000). A lo largo de la vida de las personas los hábitos motores tienden a mantenerse, ya que el cuerpo del(la) alfarero(a) adquiere sus ritmos en la emulación de las formas de hacer del maestro y difícilmente los cambiará.

$\mathrm{El}$ interés de estas consideraciones radica en la relación constatada en comunidades de alfareros 
tradicionales estudiados por la etnoarqueología, de los atributos visibles con esferas amplias de interacción y macroidentidades y de los atributos ocultos con comunidades de alfareros e identidades sociales a menor escala, como familia o grupo corresidencial (Gosselain 2000; Stark 1998). Es allí donde las tradiciones tecnológicas cobran su sentido y significados más profundos y refuerzan la identidad con quienes se comparten las prácticas sociales (Gosselain 2010).

Trabajamos con cuatro niveles operativos de organización espacial. El nivel mínimo de resolución son las concentraciones de basura producto de una o más unidades residenciales. Un segundo nivel está conformado por concentraciones contiguas dentro de un sitio arqueológico que entendemos como un conjunto corresidencial. El tercer nivel es el de la localidad, un espacio de dimensiones variadas pero acotadas (no mayor a unos 10 a 20 $\mathrm{km}$ lineales en nuestra región de estudio), separada de otras localidades por espacios sin ocupación humana o con una notoria baja en la densidad de las ocupaciones. El cuarto nivel incluye más de una localidad y/o la microrregión (supralocal).

\section{Materiales y Métodos}

Se trabajó con los materiales recuperados de sitios arqueológicos, reconocidos en una prospección de cobertura total en un área de ca. 20 x 10 km (Cornejo et al. 2012), donde se realizaron múltiples pozos de sondeo de 40 x $40 \mathrm{~cm}$, harneados y excavados hasta el suelo estéril, colectas intensivas de superficie en cuadrantes de $25 \times 25 \mathrm{~m}$ y colectas intensivas siguiendo transectas en terrenos con frutales (Figura 1). La zona de estudio es un área de intenso uso agrícola, donde los depósitos culturales han sido con notable cuantía afectados por alteraciones posdepositacionales. A consecuencia de ello no se conservan rasgos correspondientes a viviendas ni a las distintas áreas de actividad, ni en superficie ni en estratigrafía. Pese a ello, aún se distinguen zonas donde se concentran los desechos materiales así como espacios vacíos o zonas de muy baja densidad que los rodean. En cada sitio se delimitaron estas concentraciones cuantificando la densidad de la cerámica recuperada en gramos por litro, con el objetivo de separar los depósitos que eventualmente podían pertenecer a distintas ocupaciones o tener rangos cronológicos diferentes (ver Cornejo et al. 2012; Falabella, Cornejo, Correa y Sanhueza 2014 para mayores detalles metodológicos). Cada una de estas concentraciones pueden ser el resultado de la unión o superposición de varios basurales que han perdido la estructura original con el tiempo, pero que no han llegado a obliterarla por completo. A consecuencia de estos procesos, la cerámica, incluso la obtenida en los pozos de sondeo, está en extremo fragmentada y erosionada, y casi todo el estrato cultural tiene alterada su estratigrafía, pudiendo estar entremezclados los depósitos de distintas ocupaciones, lo que dificulta una buena resolución contextual. Muchos de estos contextos son, además, de baja potencia.

En la muestra están representados dos componentes culturales, Bato y Llolleo. Si bien las ocupaciones Bato son anteriores a las Llolleo (desde inicios de la era cristiana en adelante), ambos son contemporáneos al menos entre ca. 400 y 1.200 d.C. Todas las concentraciones analizadas corresponden a basuras domésticas y como tales nos remiten a un espacio residencial. Estas son nuestras unidades mínimas de análisis espacial. Cuentan con control cronológico por termoluminiscencia (ca. 2 a 4 muestras en cada una, con 152 fechados) además de ocho fechados ${ }^{14} \mathrm{C}$ que caen dentro del rango de los fechados TL del depósito correspondiente (Falabella, Cornejo, Sanhueza y Correa 2014).

Las muestras de cerámica que utilizamos para este trabajo provienen de 35 concentraciones, obtenidas de 17 sitios arqueológicos de las localidades de Valdivia de Paine (VP), Colonia Kennedy (CK) y Peuco (EP) en la microrregión de Angostura (Figura 1) (Falabella, Cornejo, Correa y Sanhueza 2014).

En arqueología existen múltiples aproximaciones a la alfarería (Rice 1987), no obstante las alternativas y posibilidades se van reduciendo a medida que los materiales y los contextos de procedencia están más alterados. Para este estudio se utilizaron solo los fragmentos gruesos y muy gruesos que en los contextos que estudiamos corresponden generalmente a "ollas" o vasijas de cocina, con el fin de no introducir ruidos si comparáramos categorías de vasijas que tienen usos diferenciados (p.ej., jarros pulidos delgados con ollas alisadas gruesas). Estas vasijas son las más abundantes en los depósitos residenciales y son las que tienen menores probabilidades de ser intercambiadas o circular por la región. Por este motivo tienen buenas posibilidades de reflejar las tradiciones de manufactura alfareras 
locales. El estudio lo abordamos por medio del análisis de los bordes y de las pastas de bordes y cuerpos, que son aquellas partes y atributos de las vasijas que se han preservado pese a las alteraciones posdepositacionales, que poseen buena representación y números susceptibles de análisis cuantitativos. En los bordes se observaron las huellas de los gestos manuales realizados con la masa en estado plástico. En las pastas buscamos indicadores de la elección de fuentes de materias primas, sus procedencias y los modos de preparar la masa. Todos ellos remiten a información sustantiva relativas a aspectos de las tradiciones tecnológicas que se transmiten cara a cara entre alfareras(os).

Dentro de cada concentración se controló que los bordes que entraron a la base de datos para este análisis, así como cada cuerpo de los analizados por petrografía y los que fueron analizados por activación neutrónica, tuviesen suficientes diferencias entre sí como para no pertenecer a una misma pieza. Las diferencias de pasta, tratamientos de superficie, formas y, en ciertos casos los colores y espesores, fueron la base para que, en términos cuantitativos, cada fragmento analizado dentro de una concentración haya sido considerado como representativo de una vasija diferente. No podemos determinar que los fragmentos de cuerpo utilizados en los análisis de petrografía y activación neutrónica sean de vasijas diferentes a los bordes analizados, pero esto no tiene implicancias para el tipo de inferencias realizadas en este trabajo.

\section{Bordes}

Este análisis incluyó una combinación de atributos cualitativos y cuantitativos. De todas las observaciones realizadas, rescatamos dos aspectos que resultan de los gestos manuales realizados cuando la pasta aún está plástica: la forma del borde-labio y las huellas de gestos técnicos de manufactura. El primero depende en gran parte del movimiento de las manos, de la posición de dedos y pulgar y el uso de instrumentos al terminar la pieza (Shepard 1980 [1954]:247); los segundos dependen de la redistribución del exceso de pasta fresca durante la manufactura.

\section{Pastas}

El estudio de las pastas incluyó tres procedimientos analíticos.
(1) Observación de un corte fresco de fragmentos de cuerpos de las vasijas alisadas de paredes gruesas del $20 \%$ del total de los fragmentos de cada concentración usando una lupa binocular bajo magnificación de 10x a 40x. Este mismo procedimiento se realizó con todos los bordes de paredes gruesas. Esta observación entregó datos sobre el tipo de áridos y granulometría a partir de los cuales se clasificó el material en patrones de pasta. El tipo de áridos dominante en las muestras según el tamaño, forma, color, frecuencia y mineralogía aparente de las inclusiones se utilizó para definir familias de pasta. El tamaño del grano se estimó con una plantilla calibrada de acuerdo con la escala de Wentworth y se utilizaron dos categorías para la distribución de tamaños, unimodal y heterogénea.

(2) Para los análisis por activación neutrónica se estudiaron 317 fragmentos de cuerpos de vasijas, gruesos y muy gruesos, y 7 muestras de potenciales materias primas arcillosas recolectadas en la zona. Estos análisis fueron realizados en el laboratorio de Arqueometría del Reactor de la Universidad de Missouri (MURR) y siguieron los protocolos estándares descritos por Glascock (1992) para la preparación, irradiación y espectroscopia. Estos análisis determinaron la concentración de 33 elementos químicos mayoritarios, minoritarios y trazas. Los datos de 32 de estos elementos, transformados al $\log 10$, se sometieron a análisis multivariados de componentes principales, análisis de cluster y distancias de Mahalanobis para agrupar muestras de perfil químico similares (Neff 2000). Todos los análisis estadísticos se realizaron con el programa Gauss elaborado y facilitado por el laboratorio de Arqueometría del MURR. Se tomaron entre 15 y 20 muestras de 18 concentraciones ( 8 de VP, 9 de CK, 1 de EP) y se seleccionaron proporcionalmente de acuerdo con las familias de pasta más frecuentes en cada una de ellas. Se prepararon ladrillos experimentales con muestras arcillosas colectadas en diferentes lugares del depósito sedimentario cuaternario en la zona de Colonia Kennedy (G13, G5, H10), tres colectadas en dos lugares de la formación riolítica Las Chilcas (Las Tazas, Lomas del Águila) y una en los intrusivos de los faldeos de los cerros de la Angostura (Figura 1). Previo a los análisis éstos se sometieron a cocción en horno eléctrico hasta alcanzar $700^{\circ} \mathrm{C}$ de temperatura.

(3) El análisis petrográfico de secciones delgadas de cerámica fue realizado por la coautora E. Fonseca. 
Tuvo un enfoque cuantitativo para ponderar la abundancia relativa de rocas y minerales de cada muestra y un enfoque cualitativo que apuntaba a asignar la combinación de inclusiones de cada muestra a una formación geológica para determinar, en lo posible, la procedencia local o foránea. Igual que para la activación neutrónica, se seleccionaron muestras de los grupos de pastas más frecuentes en las concentraciones estudiadas. Se analizaron 90 cortes petrográficos en total.

\section{Resultados}

\section{Diferenciación de tradiciones tecnológicas y su distribución espacial}

Se identificó una gran variedad de bordes y labios pero solo algunos alcanzaron frecuencias importantes. El 86\% de 1.979 bordes analizados corresponde a combinaciones entre cinco formas de borde (evertidos, rectos, evertido interior/recto exterior, evertido/abultado, recto/invertido) y cuatro de labio (plano, redondeado, plano/redondeado, redondeado oblicuo, plano/engrosado) (Tabla 1, Figura 2). La mayoría se encontró tanto en contextos Llolleo como Bato y en ambas localidades analizadas se observaron variaciones en sus frecuencias. Algunas adquieren especial relevancia al compararlas por contexto cultural. Los bordes evertido/abultado son significativamente más frecuentes en contextos Llolleo $(z=7,083 p<0,01)$ y los recto/invertido de labio plano engrosado en ocupaciones Bato $(\mathrm{z}=-5,760 \mathrm{p}<0,01)$. Creemos que, aunque las cantidades son bajas, la diferencia presentada específicamente en relación con los bordes recto invertido de labio plano engrosado apunta a tradiciones tecnológicas diferenciadas en la medida que tiene una distribución principalmente asociada y circunscrita a tres concentraciones Bato de la localidad de Valdivia de Paine (VP5/A, VP5/B y VP3/D). Estas ocupaciones tienen en común el contar con dataciones muy tempranas y presentar pastas de familia granítica heterogénea, muy similares entre sí. Estos bordes corresponden a vasijas que pueden llevar asas mamelonares (Figura 2). Otras variaciones en las frecuencias en cambio se diferencian principalmente por localidad; los bordes evertidos son notoriamente más frecuentes en Colonia Kennedy que en Valdivia de Paine ( $\mathrm{z}$ $=-9,827 p<0,01)$, tanto en Bato como en Llolleo, y los bordes evertido interior/recto exterior son en cambio más frecuentes en Valdivia de Paine, también en ambos contextos $(\mathrm{z}=3,973 \mathrm{p}<0,01)$.

Las huellas de manufactura relacionadas con gestos técnicos, si bien escasas, también mostraron algunas diferencias espaciales. Una de ellas es un ángulo marcado en los límites del labio, que se encuentra en uno (le) o ambos lados (lee) del mismo. La apariencia hace pensar en el acabado del labio mediante algún instrumento plano, en una forma particular de posicionar los dedos durante esta acción o en una combinación de ambos (Tabla 2). Este es un rasgo que destaca en los sitios Bato y sobremanera en los de la localidad Valdivia de Paine. Específicamente, el sitio VP5 es el único que tiene el $40 \%$ de los labios con esta característica (de 439 bordes analizados en ese sitio) mientras los otros no sobrepasan el $20 \%$ y la mayoría está por debajo del $10 \%$. Además, solo en VP5 el ángulo en los límites del labio ocurre tanto en los labios planos como en los labios redondeados, plano/redondeados y redondeado oblicuo. Este hecho nos hace pensar que se trata de un gesto técnico asociado a las alfareras(os) de la comunidad corresidencial VP5.

$\mathrm{Se}$ registraron también distribuciones diferenciales en el refuerzo del borde. Esta acción consiste en dar vuelta la placa de arcilla de la pared del borde en estado plástico y adherirla al mismo por el exterior dejando un engrosamiento y un desnivel que es visible por el exterior de la vasija (Figura 2). Si bien esta huella se encontró presente en ambas localidades y contextos se asocia particularmente con los sitios Llolleo de Valdivia de Paine $(\mathrm{z}=2,643$ $\mathrm{p}<0,01$ ) (Tabla 3).

Otros gestos técnicos, como el desplazamiento de greda fresca producto del movimiento de los dedos del alfarero hacia el interior o exterior del labio o las irregularidades o estriaciones sobre el labio producto del desplazamiento de greda por el uso de algún instrumento, pueden ser obliterados por las acciones destinadas a emparejar las superficies de las piezas (alisado/pulido) posteriores. Consideramos que no solo su presencia es indicativa de ciertas prácticas, sino también el hecho de no encontrarlas, en la medida que implican acciones de los alfareros. La primera de ellas tiene una representatividad muy similar en ambas localidades, aunque dejar este tipo de huellas parece ser algo menos común entre los alfareros Llolleo de la localidad de Colonia Kennedy (Tabla 4). La segunda tiene una ocurrencia mucho más frecuente en la localidad de Colonia Kennedy, tanto en Llolleo como en Bato (Tabla 5). 
Tabla 1. Frecuencia de formas de bordes y labios por localidad y contexto cultural. Rim and lip frequency according to locality and cultural context.

\begin{tabular}{|c|c|c|c|c|c|c|c|c|c|}
\hline & Formas & & onia Ken & dy & & livia de $\mathrm{F}$ & & & \\
\hline Borde-L & abio & Bato & Llolleo & Total CK & Bato & Llolleo & Total VP & & \\
\hline & Plano & 5 & 5 & 10 & 4 & 41 & 45 & 55 & 2,8 \\
\hline & Plano redondeado & 3 & 2 & 5 & 1 & 16 & 17 & 22 & 1,1 \\
\hline 을 윰 & Redondeado & 6 & 3 & 9 & 2 & 23 & 25 & 34 & 1,7 \\
\hline 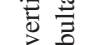 & Redondeado oblicuo & 2 & 8 & 10 & 3 & 29 & 32 & 42 & 2,1 \\
\hline & N Total & 16 & 18 & 34 & 10 & 109 & 119 & 153 & 7,7 \\
\hline & $\%$ Total & 6,5 & 7,3 & 6,9 & 2,5 & 15,5 & 10,0 & 7,7 & \\
\hline $\bar{i}$ & Plano & 6 & 7 & 13 & 27 & 33 & 60 & 73 & 3,7 \\
\hline.$\overline{0}$ & Plano redondeado & 2 & 4 & 6 & 12 & 17 & 29 & 35 & 1,8 \\
\hline$\stackrel{\bar{E}}{\Xi}$ & Redondeado & 6 & 7 & 13 & 27 & 33 & 60 & 71 & 3,6 \\
\hline $\begin{array}{ll}:= & \overline{1} \\
0 & 0 \\
0 & 0\end{array}$ & Redondeado oblicuo & 11 & 15 & 26 & 24 & 70 & 94 & 120 & 6,1 \\
\hline 过 & Total & 27 & 28 & 55 & 91 & 153 & 244 & 299 & 15,1 \\
\hline [1 & $\%$ Total & 11,0 & 11,4 & 11,2 & 17,7 & 21,7 & 20,0 & 15,1 & \\
\hline & Plano & 46 & 60 & 106 & 85 & 96 & 181 & 287 & 14,5 \\
\hline & Plano redondeado & 18 & 17 & 35 & 12 & 35 & 47 & 82 & 4,1 \\
\hline 음 & Redondeado & 35 & 47 & 82 & 36 & 38 & 74 & 156 & 7,9 \\
\hline$\stackrel{\frac{1}{2}}{>}$ & Redondeado oblicuo & 8 & 9 & 17 & 15 & 12 & 27 & 44 & 2,2 \\
\hline & Total & 107 & 133 & 240 & 148 & 181 & 329 & 569 & 28,8 \\
\hline & $\%$ Total & 43,5 & 54,3 & 48,9 & 28,8 & 25,7 & 27,0 & 28,8 & \\
\hline & Plano & 55 & 46 & 101 & 93 & 125 & 218 & 319 & 16,1 \\
\hline & Plano redondeado & 5 & 6 & 11 & 32 & 27 & 59 & 70 & 3,5 \\
\hline 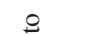 & Redondeado & 22 & 10 & 32 & 59 & 79 & 138 & 170 & 8,6 \\
\hline$\ddot{d}$ & Redondeado oblicuo & 8 & 1 & 9 & 10 & 8 & 18 & 27 & 1,4 \\
\hline & Total & 90 & 63 & 153 & 194 & 239 & 433 & 586 & 29,6 \\
\hline & $\%$ Total & 36,6 & 25,7 & 31,2 & 38,3 & 33,9 & 35,8 & 29,6 & \\
\hline 0 & Plano & 4 & 2 & 6 & 36 & 22 & 58 & 64 & 3,2 \\
\hline Oे & Plano-engrosado & 2 & 1 & 3 & 27 & 1 & 28 & 31 & 1,6 \\
\hline$\stackrel{0}{ \pm}$ & Total & 6 & 3 & 9 & 63 & 23 & 86 & 95 & 4,8 \\
\hline$-\Xi$ & $\%$ Total & 2,4 & 1,2 & 1,8 & 12,7 & 3,3 & 7,2 & 4,8 & \\
\hline$\ddot{0}$ & Otros & 36 & 44 & 80 & 94 & 103 & 197 & 277 & 14,0 \\
\hline 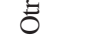 & $\%$ Total & 13 & 15 & 14,0 & 16,0 & 13,0 & 14,0 & 14,0 & \\
\hline N Total & & 282 & 289 & 571 & 600 & 808 & 1.408 & 1.979 & 100 \\
\hline$\%$ Total & & 100 & 100 & 100 & 100 & 100 & 100 & 100 & \\
\hline
\end{tabular}

Se reconocieron también tradiciones tecnológicas en la preparación de pastas. Se identificaron seis grandes familias, todas con temperante de arena, que muestran dos tendencias generalizadas en los sitios analizados: unidades donde predominan las pastas con áridos volcánicos $(\mathrm{V}, \mathrm{VB})$ con granulometría unimodal y unidades con predominio de pastas con áridos graníticos (Gr, Grn) con granulometría heterogénea (Tablas 6 y 7). La diferencia se correlaciona con el contexto cultural: Llolleo en el primer caso, y Bato en el segundo. Esta tendencia no es algo nuevo dentro de Chile Central. Estudios de pasta anteriores en sitios tanto de los valles interiores como de zonas costeras había tenido resultados similares (Sanhueza 2004). Estos datos indican que la tendencia trasciende la microrregión de Angostura y es una tradición tecnológica de escala regional.

\section{Distribución de recursos y fuentes de materias primas}

Uno de los resultados más robustos en relación con la procedencia de materias primas lo obtuvimos 


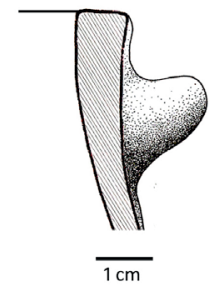

Borde recto invertido Labio plano engrosado

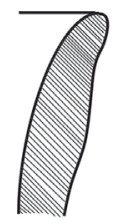

$\overline{1 \mathrm{~cm}}$

Borde evertido/abultado Labio redondeado oblicuo

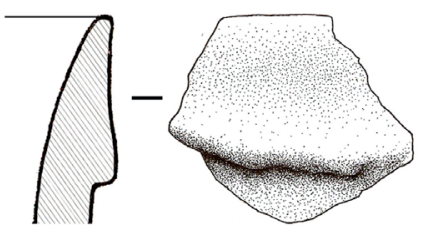

$\overline{1 \mathrm{~cm}}$

Borde reforzado

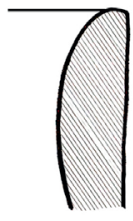

$1 \mathrm{~cm}$

Borde evertido interior/ recto exterior Labio redondeado oblicuo
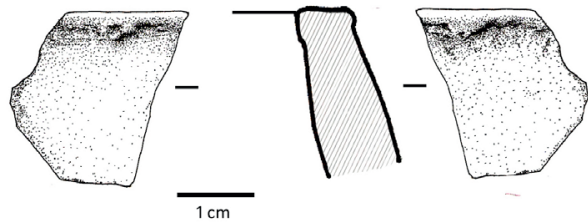

Desplazamiento de greda interior y exterior
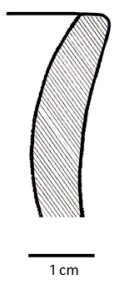

Borde evertido Labio plano

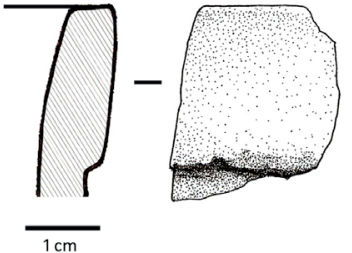

Borde reforzado

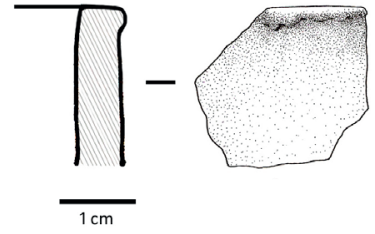

Desplazamiento de greda exterior

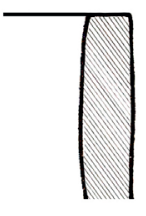

$\overline{1 \mathrm{~cm}}$

Borde recto Labio plano

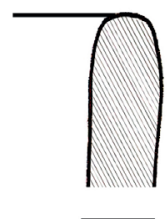

$\overline{1 \mathrm{~cm}}$

Borde recto Labio redondeado

Figura 2. Formas de bordes y labios frecuentes en la muestra estudiada.

Main rim and lip types in the archaeological sites from Angostura micro region.

Tabla 2. Bordes sin ángulo (1), con ángulo en uno (le) y dos (lee) lados del labio.

Rims without a corner point (l), with a corner point in one (le) or two (lee) sides of the lip.

\begin{tabular}{|c|c|c|c|c|c|c|c|}
\hline \multirow{2}{*}{ Ángulo en labio(s) } & \multicolumn{3}{|c|}{ Colonia Kennedy } & \multicolumn{3}{|c|}{ Valdivia de Paine } & \multirow{2}{*}{ N Total } \\
\hline & Bato & Llolleo & Total CK & Bato & Llolleo & Total VP & \\
\hline 1 & $90,7 \%$ & $95,8 \%$ & $93,3 \%$ & $67,8 \%$ & $89,2 \%$ & $80,1 \%$ & 1.652 \\
\hline le & $6,4 \%$ & $2,1 \%$ & $4,2 \%$ & $14,4 \%$ & $7,1 \%$ & $10,2 \%$ & 167 \\
\hline lee & $2,8 \%$ & $2,1 \%$ & $2,5 \%$ & $17,8 \%$ & $3,7 \%$ & $9,7 \%$ & 150 \\
\hline Total & $100 \%$ & $100 \%$ & $100 \%$ & $100 \%$ & $100 \%$ & $100 \%$ & \\
\hline $\mathrm{N}$ total & 281 & 285 & 566 & 596 & 807 & 1.403 & 1.969 \\
\hline
\end{tabular}

de los análisis por activación neutrónica. Los resultados del análisis de componentes principales y de las distancias de Mahalanobis - que consideró los primeros ocho factores y $88 \%$ de la varianza-, formaron cinco grupos químicos para los fragmentos cerámicos de la microrregión de Angostura $^{1}$ (Figura 3, Tablas 8 y 9).
El grupo 1 es particular por un alto contenido de Cr. Es exclusivo de la localidad de Valdivia de Paine, particularmente de los sitios VP2 y VP3 cercanos al río Maipo y se caracteriza por las pastas volcánicas (V y VB). El grupo 2 también es propio de Valdivia de Paine, pero contiene pastas graníticas (Gr), además de las anteriores. Incluye muestras de 
Tabla 3. Porcentaje de bordes reforzados.

Frequency of reinforced rims.

\begin{tabular}{|c|c|c|c|c|c|c|c|}
\hline \multirow{2}{*}{ Borde reforzado } & \multicolumn{3}{|c|}{ Colonia Kennedy } & \multicolumn{3}{|c|}{ Valdivia de Paine } & \multirow{2}{*}{ N Total } \\
\hline & Bato & Llolleo & Total CK & Bato & Llolleo & Total VP & \\
\hline Con refuerzo & $3,5 \%$ & $2,1 \%$ & $2,8 \%$ & $3,1 \%$ & $7,6 \%$ & $5,7 \%$ & 95 \\
\hline Sin refuerzo & $96,5 \%$ & $97,9 \%$ & $97,2 \%$ & $96,9 \%$ & $92,4 \%$ & $94,3 \%$ & 1.864 \\
\hline Total & $100 \%$ & $100 \%$ & $100 \%$ & $100 \%$ & $100 \%$ & $100 \%$ & \\
\hline N Total & 282 & 289 & 571 & 587 & 801 & 1.388 & 1.959 \\
\hline
\end{tabular}

Tabla 4. Porcentaje de bordes con huella del desplazamiento de greda hacia el exterior e interior. Rims with excess clay on the outer or inner edges.

\begin{tabular}{|c|c|c|c|c|c|c|c|}
\hline \multirow{2}{*}{ Huella de greda } & \multicolumn{3}{|c|}{ Colonia Kennedy } & \multicolumn{3}{|c|}{ Valdivia de Paine } & \multirow{2}{*}{ N Tota } \\
\hline & Bato & Llolleo & Total Ck & Bato & Llolleo & Total Vp & \\
\hline Exterior & $24,8 \%$ & $17,3 \%$ & $21,0 \%$ & $23,5 \%$ & $25,2 \%$ & $24,5 \%$ & 465 \\
\hline Ext. e int. & $0,7 \%$ & $1,0 \%$ & $0,9 \%$ & $1,5 \%$ & $0,0 \%$ & $0,6 \%$ & 14 \\
\hline Interior & $3,9 \%$ & $1,7 \%$ & $2,8 \%$ & $1,5 \%$ & $3,0 \%$ & $2,3 \%$ & 49 \\
\hline Sin huella & $70,6 \%$ & $79,9 \%$ & $75,3 \%$ & $73,5 \%$ & $71,8 \%$ & $72,5 \%$ & 1.450 \\
\hline Total & $100 \%$ & $100 \%$ & $100 \%$ & $100 \%$ & $100 \%$ & $100 \%$ & \\
\hline N Total & 282 & 289 & 571 & 599 & 808 & 1.407 & 1.978 \\
\hline
\end{tabular}

Tabla 5. Porcentaje de irregularidades sobre el labio.

Frequency of irregular lip finishing.

\begin{tabular}{|c|c|c|c|c|c|c|c|}
\hline \multirow{2}{*}{ Irregularidad en labio } & \multicolumn{3}{|c|}{ Colonia Kennedy } & \multicolumn{3}{|c|}{ Valdivia de Paine } & \multirow{2}{*}{ N Total } \\
\hline & Bato & Llolleo & Total CK & Bato & Llolleo & Total VP & \\
\hline Con irreg. & $16,7 \%$ & $23,9 \%$ & $20,3 \%$ & $11,2 \%$ & $15,8 \%$ & $13,9 \%$ & 311 \\
\hline Sin irreg. & $83,3 \%$ & $76,1 \%$ & $79,7 \%$ & $88,8 \%$ & $84,2 \%$ & $86,1 \%$ & 1.667 \\
\hline Total & $100 \%$ & $100 \%$ & $100 \%$ & $100 \%$ & $100 \%$ & $100 \%$ & \\
\hline N Total & 282 & 289 & 571 & 599 & 808 & 1.407 & 1.978 \\
\hline
\end{tabular}

Tabla 6. Familias de pasta. Paste groups.

\begin{tabular}{|c|c|c|c|c|c|c|c|}
\hline \multirow{2}{*}{ Familia de Pasta } & \multicolumn{3}{|c|}{ Colonia Kennedy } & \multicolumn{3}{|c|}{ Valdivia de Paine } & \multirow{2}{*}{ N Tota } \\
\hline & Bato & Llolleo & Total CK & Bato & Llolleo & Total VP & \\
\hline $\mathrm{Gr}$ & $49,6 \%$ & $15,9 \%$ & $32,9 \%$ & $37,9 \%$ & $33,5 \%$ & $35,4 \%$ & 517 \\
\hline Grn & $9,9 \%$ & $1,6 \%$ & $5,8 \%$ & $7,8 \%$ & $0,4 \%$ & $3,6 \%$ & 65 \\
\hline $\mathrm{HB}$ & $0,0 \%$ & $0,0 \%$ & $0,0 \%$ & $1,1 \%$ & $5,3 \%$ & $3,5 \%$ & 35 \\
\hline Otro & $1,6 \%$ & $2,0 \%$ & $1,8 \%$ & $0,7 \%$ & $2,0 \%$ & $1,4 \%$ & 23 \\
\hline $\mathrm{V}$ & $4,8 \%$ & $3,3 \%$ & $4,0 \%$ & $8,3 \%$ & $1,8 \%$ & $4,6 \%$ & 66 \\
\hline VB & $20,2 \%$ & $31,3 \%$ & $25,7 \%$ & $26,2 \%$ & $51,8 \%$ & $40,6 \%$ & 533 \\
\hline VGr & $13,9 \%$ & $45,9 \%$ & $29,7 \%$ & $17,9 \%$ & $5,3 \%$ & $10,8 \%$ & 256 \\
\hline Total & $100 \%$ & $100 \%$ & $100 \%$ & $100 \%$ & $100 \%$ & $100 \%$ & \\
\hline N Total & 252 & 246 & 498 & 435 & 562 & 997 & 1.495 \\
\hline
\end{tabular}


Tabla 7. Granulometría.

Grain size distribution.

\begin{tabular}{|c|c|c|c|c|c|c|c|}
\hline \multirow{2}{*}{ Granulometría } & \multicolumn{3}{|c|}{ Colonia Kennedy } & \multicolumn{3}{|c|}{ Valdivia de Paine } & \multirow{2}{*}{ N Tota } \\
\hline & Bato & Llolleo & Total CK & Bato & Llolleo & Total VP & \\
\hline Unimodal & $65,3 \%$ & $86,3 \%$ & $75,6 \%$ & $76,0 \%$ & $84,4 \%$ & $80,7 \%$ & 1.142 \\
\hline Heterogénea & $34,7 \%$ & $13,7 \%$ & $24,4 \%$ & $24,0 \%$ & $15,6 \%$ & $19,3 \%$ & 304 \\
\hline Total & $100 \%$ & $100 \%$ & $100 \%$ & $100 \%$ & $100 \%$ & $100 \%$ & \\
\hline N Total & 245 & 234 & 479 & 430 & 537 & 967 & 1.446 \\
\hline
\end{tabular}

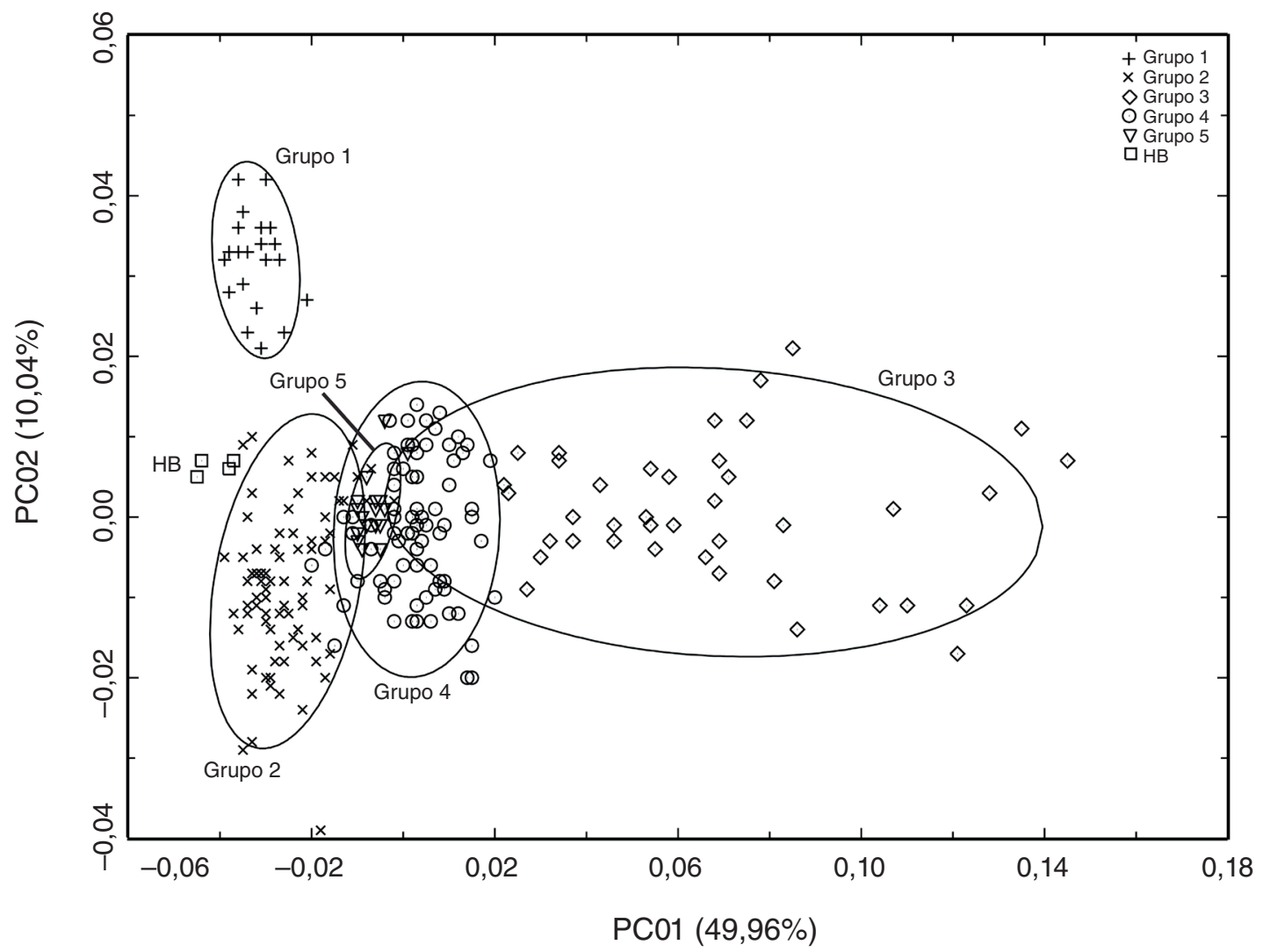

Figura 3. Gráfico de los componentes principales PC01 y PC02 con los cinco grupos químicos. Las elipses representan intervalos de confianza de $90 \%$ para la inclusión de las muestras en cada grupo.

A principal component plot showing the five compositional groups. The ellipses represent $90 \%$ confidence intervals for membership in each of the groups.

todos los sitios de Valdivia de Paine y solo algunas $(n=12)$ de Colonia Kennedy. Cuatro muestras de pasta HB, que es propia del sitio VP1, forman un pequeño conjunto en los gráficos, pero no pueden considerarse un grupo químico propiamente tal.

Los grupos 3, 4 y 5 dominan en Colonia Kennedy. Muchas muestras, si bien se asignan a uno de estos grupos, también tienen probabilidades mayores a un
$10 \%$ de pertenecer a otro de ellos. El grupo 3 tiene alto contenido de $\mathrm{Sc}, \mathrm{Ca}, \mathrm{Fe}$ y baja concentración de tierras raras y metales alcalinos. Es característico del sitio Bato CK1/7 y de las pastas Grn. Todas las muestras con esta pasta, que fueron asignadas a un grupo químico, pertenecen a él. Los grupos 4 y 5 se diferencian entre sí fundamentalmente por las concentraciones de $\mathrm{Sc}, \mathrm{Na}, \mathrm{Sr}$, lo que se refleja en su 
Tabla 8. Eigenvalores, varianza explicada y coeficientes de los elementos de los primeros ocho factores del análisis de componentes principales R-Q simultáneo, basado en matriz de varianza-covarianza. Valores más importantes en negrita. Eigenvalues, variance explained, and element coefficients for the first eight factors of the simultaneous $R$ - $Q$ factor analysis based on variance-covariance matrix. The most important values are in bold.

\begin{tabular}{|c|c|c|c|c|c|c|c|c|}
\hline Factores & PC01 & $\mathrm{PC} 02$ & $\mathrm{PC} 03$ & PC04 & PC05 & PC06 & PC07 & PC08 \\
\hline Eigenvalor & 0,369 & 0,074 & 0,052 & 0,043 & 0,039 & 0,033 & 0,020 & 0,017 \\
\hline$\%$ Varianza & 49,96 & 10,04 & 7,04 & 5,80 & 5,32 & 4,41 & 2,65 & 2,33 \\
\hline $\begin{array}{l}\% \text { Varianza } \\
\text { acumulada }\end{array}$ & 49,96 & 60,00 & 67,04 & 72,84 & 78,17 & 82,57 & 85,22 & 87,56 \\
\hline As & $-0,008$ & 0,034 & 0,697 & $-0,012$ & $-0,241$ & $-0,068$ & $-0,069$ & 0,579 \\
\hline $\mathrm{La}$ & $-0,197$ & 0,012 & $-0,025$ & $-0,213$ & $-0,077$ & $-0,037$ & 0,089 & $-0,060$ \\
\hline $\mathrm{Lu}$ & $-0,066$ & $-0,051$ & 0,058 & $-0,184$ & 0,027 & 0,164 & 0,056 & $-0,038$ \\
\hline $\mathrm{Nd}$ & $-0,150$ & 0,032 & $-0,013$ & $-0,271$ & $-0,098$ & $-0,009$ & 0,053 & $-0,009$ \\
\hline $\mathrm{Sm}$ & $-0,101$ & 0,035 & 0,023 & $-0,275$ & $-0,030$ & 0,059 & $-0,006$ & $-0,054$ \\
\hline $\mathrm{U}$ & $-0,278$ & $-0,061$ & 0,031 & $-0,043$ & $-0,063$ & $-0,034$ & 0,272 & 0,211 \\
\hline $\mathrm{Yb}$ & $-0,049$ & $-0,033$ & 0,044 & $-0,217$ & 0,010 & 0,153 & 0,026 & $-0,033$ \\
\hline $\mathrm{Ce}$ & $-0,213$ & 0,013 & 0,003 & $-0,204$ & $-0,119$ & $-0,026$ & 0,083 & $-0,051$ \\
\hline Co & 0,073 & 0,183 & $-0,013$ & $-0,044$ & $-0,246$ & 0,128 & $-0,206$ & $-0,032$ \\
\hline $\mathrm{Cr}$ & $-0,323$ & 0,866 & $-0,027$ & 0,175 & 0,271 & 0,018 & $-0,002$ & 0,060 \\
\hline Cs & $-0,311$ & $-0,183$ & 0,286 & 0,205 & $-0,066$ & $-0,025$ & $-0,313$ & $-0,515$ \\
\hline $\mathrm{Eu}$ & $-0,026$ & 0,070 & 0,032 & $-0,268$ & $-0,028$ & 0,048 & $-0,087$ & $-0,051$ \\
\hline $\mathrm{Fe}$ & 0,075 & 0,038 & 0,048 & $-0,089$ & $-0,042$ & 0,183 & $-0,058$ & 0,051 \\
\hline Hf & $-0,212$ & $-0,053$ & 0,070 & $-0,142$ & 0,018 & $-0,024$ & 0,115 & $-0,027$ \\
\hline $\mathrm{Rb}$ & $-0,338$ & $-0,106$ & $-0,195$ & 0,165 & $-0,166$ & 0,129 & $-0,160$ & 0,051 \\
\hline $\mathrm{Sb}$ & $-0,226$ & $-0,054$ & 0,455 & 0,154 & 0,238 & 0,145 & $-0,237$ & $-0,182$ \\
\hline $\mathrm{Sc}$ & 0,100 & 0,077 & $-0,028$ & $-0,082$ & $-0,030$ & 0,297 & $-0,115$ & 0,027 \\
\hline $\mathrm{Sr}$ & 0,026 & 0,083 & $-0,065$ & $-0,146$ & $-0,206$ & $-0,485$ & $-0,282$ & 0,068 \\
\hline $\mathrm{Ta}$ & $-0,242$ & $-0,046$ & 0,001 & $-0,128$ & $-0,069$ & $-0,139$ & 0,170 & $-0,012$ \\
\hline $\mathrm{Tb}$ & $-0,033$ & 0,025 & $-0,007$ & $-0,336$ & $-0,029$ & 0,103 & $-0,128$ & $-0,034$ \\
\hline Th & $-0,318$ & $-0,041$ & $-0,062$ & $-0,050$ & $-0,120$ & 0,016 & 0,182 & 0,045 \\
\hline $\mathrm{Zn}$ & $-0,019$ & 0,097 & $-0,088$ & 0,016 & $-0,286$ & 0,200 & $-0,067$ & $-0,086$ \\
\hline $\mathrm{Zr}$ & $-0,176$ & $-0,020$ & 0,001 & $-0,110$ & $-0,007$ & $-0,021$ & 0,173 & $-0,021$ \\
\hline $\mathrm{Al}$ & 0,020 & $-0,006$ & $-0,019$ & $-0,005$ & 0,013 & 0,041 & $-0,041$ & 0,012 \\
\hline $\mathrm{Ba}$ & $-0,200$ & $-0,134$ & $-0,254$ & 0,011 & 0,080 & $-0,132$ & $-0,465$ & 0,373 \\
\hline $\mathrm{Ca}$ & 0,176 & 0,127 & 0,038 & $-0,205$ & $-0,020$ & $-0,317$ & $-0,238$ & $-0,157$ \\
\hline Dy & $-0,063$ & $-0,042$ & 0,003 & $-0,379$ & 0,315 & 0,132 & $-0,148$ & $-0,017$ \\
\hline $\mathrm{K}$ & $-0,282$ & $-0,137$ & $-0,278$ & 0,135 & $-0,079$ & 0,055 & $-0,259$ & 0,187 \\
\hline $\mathrm{Mn}$ & 0,045 & 0,187 & 0,038 & 0,003 & $-0,554$ & 0,195 & $-0,052$ & $-0,145$ \\
\hline $\mathrm{Na}$ & $-0,003$ & 0,143 & 0,025 & $-0,165$ & $-0,005$ & $-0,359$ & $-0,111$ & $-0,186$ \\
\hline $\mathrm{Ti}$ & 0,035 & $-0,069$ & 0,048 & $-0,213$ & $\mathbf{0 , 3 3 3}$ & 0,163 & $-0,189$ & 0,129 \\
\hline V & 0,122 & 0,086 & $-0,076$ & $-0,084$ & $-0,083$ & 0,346 & $-0,183$ & 0,098 \\
\hline
\end{tabular}

separación en el eje del factor 6 (PC06) (Figura 4). El grupo 4 es claramente el perfil de los sitios Llolleo del sureste de Colonia Kennedy y Peuco (CK1/6, CK10, CK11, EP) y el grupo 5 de los sitios de más al norte cercanos al estero La Berlina, tanto Bato como Llolleo (CK2, CK3, CK4).

Estos datos señalan que los grupos químicos se separan según localidad y no siempre por contexto cultural (Tabla 9). Dentro de cada localidad se advierte una tendencia a que las muestras de concentraciones cercanas se agrupen, lo que sugiere que los alfareros obtenían los recursos para la manufactura cerámica de fuentes cercanas a los lugares de residencia.

Los análisis de los ladrillos experimentales y petrografía también avalan el uso de fuentes locales. Las distancias de Mahalanobis de las muestras arcillosas, calculadas desde los primeros ocho factores ( $88 \%$ de varianza), se proyectaron a los cinco grupos químicos de la cerámica. Los resultados indican que las muestras G5 y G13, ambas de los depósitos arcillosos de la cuenca sedimentaria aluvial de la zona de Colonia Kennedy, tienen probabilidades de $30,4 \%$ y $48,8 \%$, respectivamente, 
Tabla 9. Muestras analizadas por activación neutrónica por sitio, asignadas a cada grupo químico. S/a son muestras sin asignar a un grupo químico.

Neutron Activation samples by site included in each chemical group. S/a are non assigned samples.

\begin{tabular}{|c|c|c|c|c|c|c|c|c|c|c|}
\hline & & Sitio & Grupo 1 & Grupo 2 & $\mathrm{HB}$ & Grupo 3 & Grupo 4 & Grupo 5 & $\mathrm{~S} / \mathrm{a}$ & Total \\
\hline \multirow{10}{*}{ 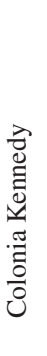 } & \multirow[t]{3}{*}{ Bato } & CK1/7 & & 1 & & 15 & & & 4 & 20 \\
\hline & & CK2/A & & 2 & & 3 & 3 & 6 & 1 & 15 \\
\hline & & CK3/B2 & & 2 & & 8 & 2 & & 3 & 15 \\
\hline & \multirow[t]{6}{*}{ Llolleo } & CK1/6 & & 2 & & & 12 & 1 & 5 & 20 \\
\hline & & CK10 & & 3 & & & 10 & & 8 & 21 \\
\hline & & CK11 & & 2 & & 1 & 13 & & 5 & 21 \\
\hline & & $\mathrm{CK} 2 / \mathrm{B}$ & & & & 2 & 2 & 7 & 4 & 15 \\
\hline & & CK3/B1 & & & & 5 & 2 & 4 & 4 & 15 \\
\hline & & $\mathrm{EP}$ & & & & & 20 & & & 20 \\
\hline & PAT & CK4 & & & & 5 & 7 & 1 & 3 & 16 \\
\hline \multirow{13}{*}{ 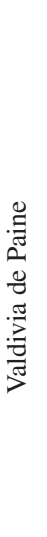 } & \multicolumn{2}{|c|}{ N Total en CK } & & 12 & 0 & 39 & 71 & 19 & 37 & 178 \\
\hline & \multicolumn{2}{|c|}{$\%$ Total en CK } & 0 & 14,5 & & 97,5 & 93,4 & 95,0 & 51,4 & \\
\hline & \multirow[t]{2}{*}{ Bato } & $\mathrm{VP} 3 / \mathrm{C}$ & & 10 & & & 1 & 1 & 2 & 14 \\
\hline & & VP3/D & 2 & 3 & & 1 & 2 & & 7 & 15 \\
\hline & \multirow{6}{*}{ Llolleo } & VP5/A & 1 & 12 & & & & & 7 & 20 \\
\hline & & VP1/AB & 1 & 14 & 4 & & & & 1 & 20 \\
\hline & & VP2 & 11 & 4 & & & 2 & & 3 & 20 \\
\hline & & $\mathrm{VP} 3 / \mathrm{A}$ & 4 & 7 & & & & & 8 & 19 \\
\hline & & $\mathrm{VP} 3 / \mathrm{B}$ & 3 & 5 & & & & & 3 & 11 \\
\hline & & VP4 & & 16 & & & & & 4 & 20 \\
\hline & \multicolumn{2}{|c|}{ N Total en VP } & 22 & 71 & 4 & 1 & 5 & 1 & 35 & 139 \\
\hline & \multicolumn{2}{|c|}{$\%$ Total en VP } & 100 & 85,5 & & 2,5 & 6,6 & 5,0 & 48,6 & \\
\hline & \multicolumn{2}{|c|}{ Total general } & 22 & 83 & 4 & 40 & 76 & 20 & 72 & 317 \\
\hline
\end{tabular}

de pertenecer al grupo químico 4 y de 20,9\% y $27,4 \%$, respectivamente, al grupo 3 . Por otra parte, tienen nula probabilidad de pertenecer a cualquiera de los otros grupos químicos (Tabla 10, Figura 4). Debido a que estas materias primas arcillosas son de textura extremadamente fina, tendrían que haber sido mezcladas con arenas para producir pastas como las de las muestras analizadas, dificultando una mayor coincidencia química. Las muestras de Angostura, cercanas al sitio El Peuco (EP), las de Lomas del Águila, cercanas a los sitios CK10 y CK11 y la de Las Tazas, cercanas a los sitios de Valdivia de Paine, no tienen probabilidad estadística de ser parte de ningún grupo químico de las muestras de cerámica analizadas. Todas ellas fueron obtenidas de formaciones volcánicas o graníticas en los cerros aledaños (Figura 1). Falta muestrear potenciales fuentes de materias primas en los depósitos arcillosos sedimentarios de la localidad de Valdivia de Paine.

La petrografía muestra que las familias de pastas tienen asociaciones mineralógicas coherentes con al menos cuatro unidades geológicas de la microrregión de Angostura: volcánico intermedio (olma), volcánico ácido (kilc), intrusivo intermedio (olmh) e intrusivo ácido (ksg, kdp) (Figura 1). Dos evidencias entregan datos más precisos con relación al uso de recursos locales. Por una parte, la presencia de granitos de textura gráfica en más del 50\% de las muestras (Figura 5). Estos han sido descritos en las formaciones geológicas de Angostura (Sellés y Gana 2001) y están presentes en las arenas colectadas en el estero Escorial y algo menos en el estero El Ajial y el río Angostura. Por otra parte, la pasta Grn se caracteriza por contener abundantes anfíbolas y/o piroxenos, plagioclasas y escaso cuarzo, asociación mineralógica que está presente en los diques de dioritas de los intrusivos del Mioceno de la formación Abanico de la cordillera de los Andes (olmh). Estas pastas prevalecen especialmente en dos concentraciones, CK1/7 y CK9/A y B en el sector de Colonia Kennedy y tienen una presencia muy escasa en las unidades Bato de Valdivia de Paine. 


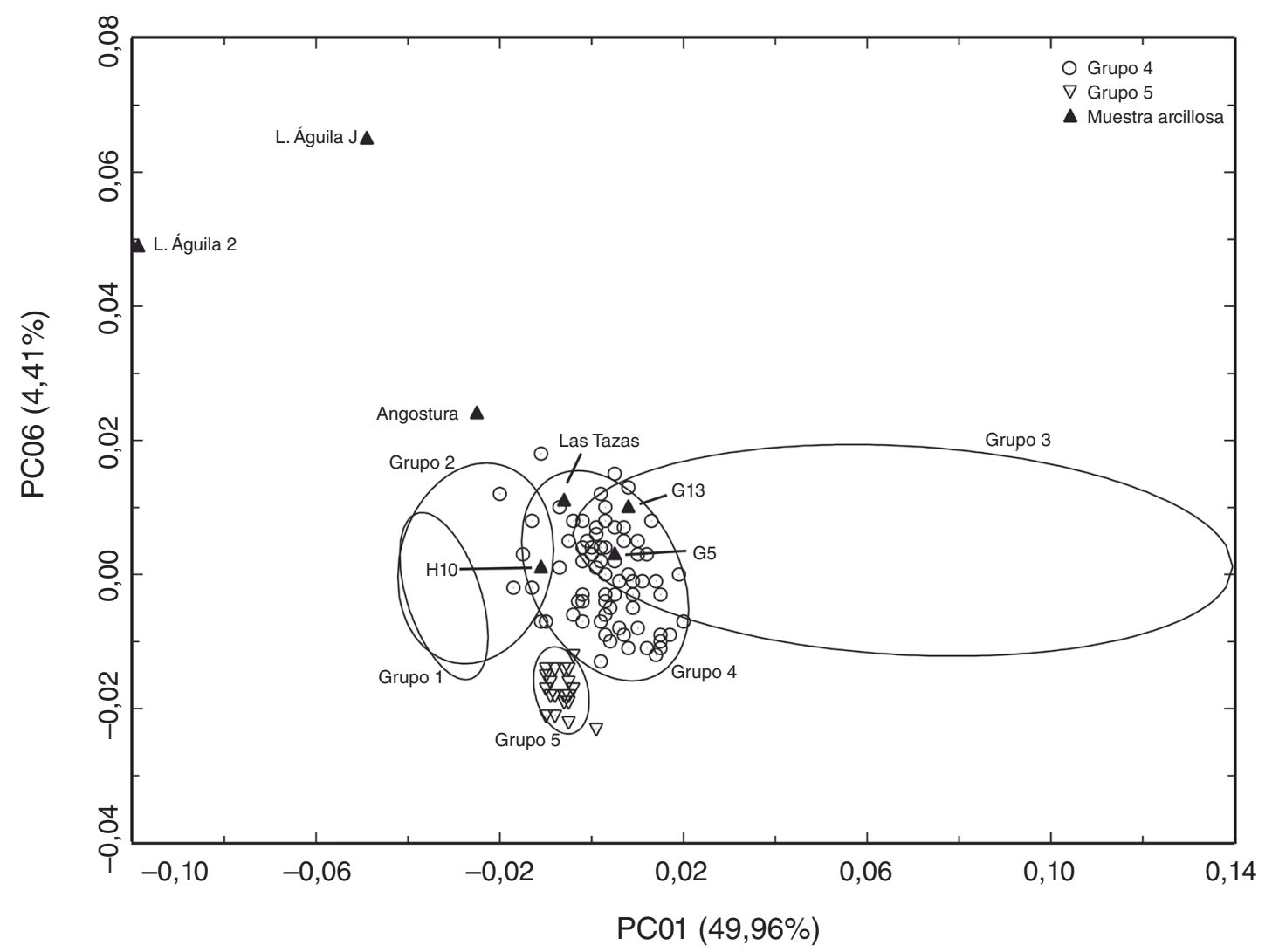

Figura 4. Gráfico de los componentes principales PC01 y PC06 que muestra la separación entre los grupos químicos 4 y 5 en el eje del PC06 y la posición de las muestras de arcillas en el espacio composicional en relación con los grupos químicos. Las elipses representan intervalos de confianza de $90 \%$ de los cinco grupos químicos de la Figura 3.

Bivariate plot of principal components PCO1 and PCO6 showing the separation between chemical groups 4 and 5 on the PCO6 axis, and the position of clay samples in the compositional space. The ellipses represent $90 \%$ confidence intervals for the five chemical groups shown in Figure 3.

Tabla 10. Probabilidad estadística de pertenencia de las muestras arcillosas a los grupos químicos de la cerámica, calculada con Distancia de Mahalanobis (8 factores, $88 \%$ de la varianza).

Mahalanobis Distance calculation of probabilities for clay sample inclusion in ceramic chemical groups.

\begin{tabular}{lccrrc}
\hline Arcillas & Grupo 1 & Grupo 2 & Grupo 3 & Grupo 4 & Grupo 5 \\
\hline Las Tazas & 0,000 & 0,000 & 0,000 & 0,000 & 0,000 \\
L. Águila J & 0,000 & 0,000 & 0,000 & 0,000 & 0,000 \\
L. Águila 2 & 0,000 & 0,000 & 0,000 & 0,000 & 0,000 \\
G5 & 0,000 & 0,000 & 20,931 & 30,399 & 0,000 \\
G13 & 0,000 & 0,000 & 27,357 & 48,818 & 0,000 \\
H10 & 0,004 & 0,000 & 0,057 & 0,149 & 0,000 \\
Angostura & 0,003 & 0,009 & 0,097 & 0,000 & 0,000 \\
\hline
\end{tabular}

\section{Discusión: Circulación de Saberes en la Microrregión de Angostura}

Las evidencias presentadas permiten generar propuestas en relación con la organización de la producción de vasijas de cocina, en cuanto a la circulación de los modos de hacer entre alfareras(os) dentro de la microrregión de Angostura. Estas evidencias tienen que ponderarse dentro de las limitaciones del material de estudio, es decir, los 

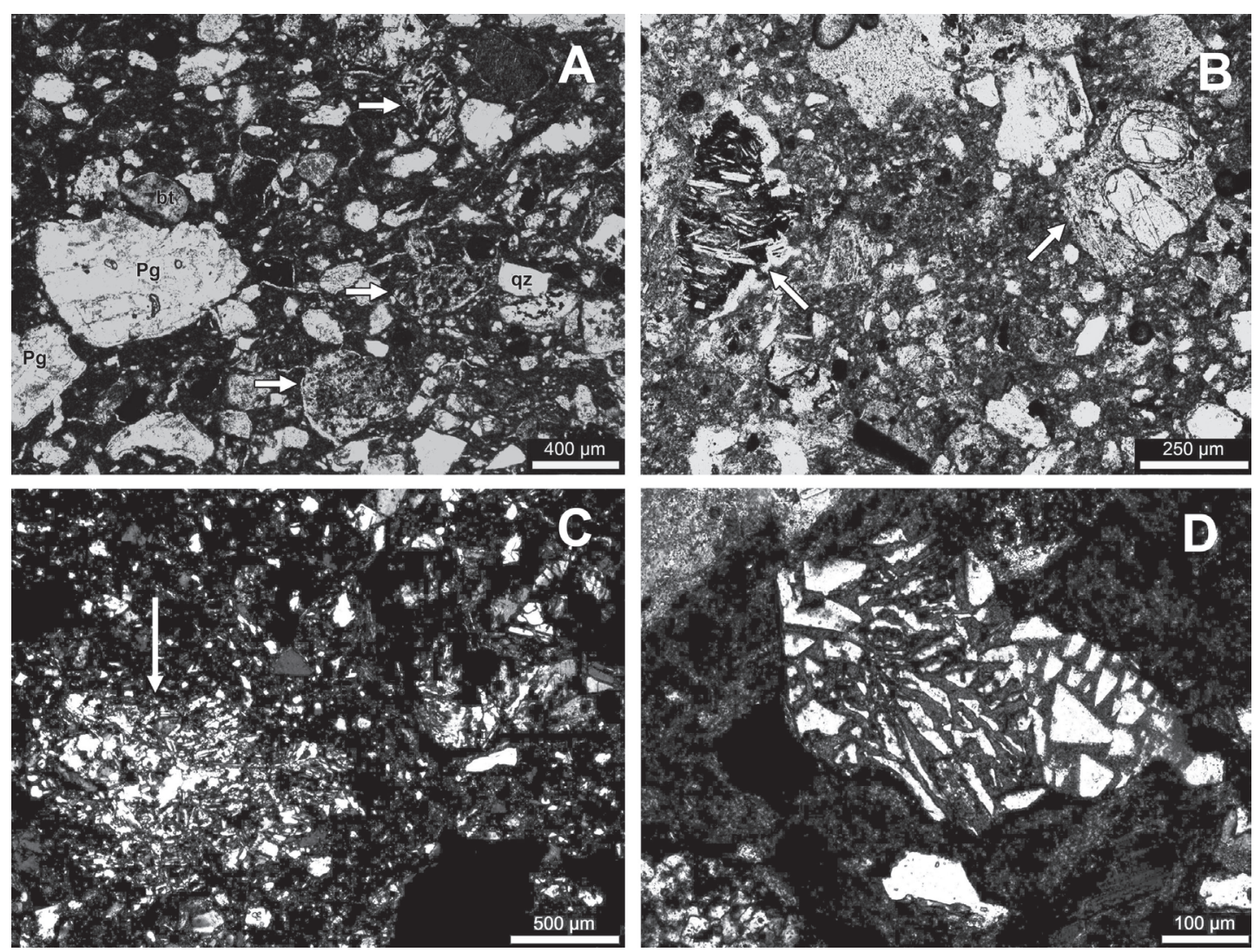

Figura 5. Microfotografías de secciones delgadas de cerámica de diferentes familias de pasta y formaciones geológicas. (A) Pasta VB, flechas blancas señalan rocas volcánicas intermedias (andesitas) (nicoles paralelos). (B) Pasta V, flechas blancas señalan rocas volcánicas de textura porfídica (andesita-dacita) (nicoles paralelos). (C). Pasta Grn, roca intrusiva (microdiorita) y otra granítica con biotita y piroxeno (nicoles cruzados). (D) Pasta Gr, feldespato potásico de roca granítica de textura gráfica (nicoles cruzados). Thin-section photomicrographs of ceramic samples from different paste groups, and geological compositions. (A) Paste VB, white arrows show intermediate volcanic rocks (andesite) (parallel nicols). (B) Paste V, white arrow shows volcanic rock with porphyritic texture (andesite-dacite) (parallel nicols). (C) Paste Grn, intrusive rock (microdiorite) and granite grain with biotite and pyroxene (crossed nicols). (D) Paste Gr, k-feldspar from a granite grain with graphic texture (crossed nicols).

escasos atributos aún visibles en los fragmentos cerámicos, los depósitos alterados y el amplio rango cronológico de la muestra.

Los resultados indican que solo unas pocas evidencias podrían validar la reproducción de modos de hacer exclusivamente a nivel del conjunto residencial. Un caso es el de VP5, por el gesto técnico que deja un ángulo pronunciado en los labios; el otro caso es el de VP1 que utiliza materias primas distintivas a la lupa y en el perfil químico (pasta HB) y eventualmente la direccionalidad del acabado del borde. En el resto de los casos los modos de hacer tienen una dispersión espacial más amplia, que sugiere que los estilos tecnológicos y las fuentes de materias primas se comparten más allá del nivel corresidencial.
Uno de estos niveles es el de la comunidad local, conjuntos de personas de distintas unidades domésticas que interactúan de forma regular en sus actividades cotidianas, que viven bastante cerca como para mantener contactos cara a cara regulares (Peterson y Drennan 2005). Nos inclinamos a pensar que cada unidad residencial o conjunto residencial contaba con alfareras(os) para el autoabastecimiento de la vajilla doméstica, pero las dinámicas de relaciones sociales en este tipo de sociedades implica la circulación de personas principalmente mediante el establecimiento de relaciones de parentesco por medio del matrimonio, y por ende de prácticas y saberes más allá de la unidad residencial de origen. Desde la perspectiva de los artesanos, se trataría de una comunidad de prácticas, entendida como personas 
que desarrollan su oficio en conjunto, compartiendo las formas de hacer (Lave y Wenger 1991).

Tanto en el caso Llolleo como Bato las diferencias que muestran los resultados entre las localidades de Valdivia de Paine y Colonia KennedyPeuco validan un sistema de interacción social intralocalidad con circulación activa de saberes al interior de cada una de ellas. Los artesanos de estas dos localidades usan materias primas diferentes y realizan opciones acerca de formas y procedimientos de terminación de las vasijas aunque no excluyentes, sí con énfasis distintos.

$\mathrm{Al}$ interior de cada una de estas localidades es muy sugerente que encontremos una cantidad de atributos, relacionados a modos de hacer, como las materias primas, las formas de bordes y labios, las irregularidades del labio, incluso la direccionalidad del acabado de bordes, que cruzan fronteras socioculturales.

Las muestras tanto de contextos Bato como Llolleo comparten, de hecho, perfiles químicos dentro de cada localidad. En la escala microespacial que cubre nuestro trabajo, los alfareros de casi todos los asentamientos podrían haber tenido acceso al mismo rango de fuentes de materias primas, al menos dentro de cada localidad. Los datos no permiten saber si las alfareras(os) utilizaban distintas fuentes locales de materias primas químicamente similares o si de modo directo compartían el uso de las mismas fuentes, pero este patrón de aprovisionamiento de materias primas a corta distancia de los sitios habitacionales es coherente con la propuesta de Arnold (1985) a partir de un amplio espectro de grupos alfareros etnográficos. Los resultados de la petrografía indican, por otro lado, que a pesar de compartir perfiles químicos al interior de cada localidad, las preferencias por los áridos que probablemente se le están agregando a la arcilla son diferentes entre Bato y Llolleo, manifestación de un estilo tecnológico singular a cada uno.

Las similitudes en relación con la forma de bordes y labio y gestos técnicos, donde en ocasiones los dos contextos culturales son más homogéneos intralocalidad que el mismo contexto entre ambas localidades, alude, sin embargo, a la posibilidad de que hayan existido ciertas prácticas compartidas, posibilitadas por la circulación de personas y sus saberes entre ambos contextos culturales. Ciertamente, esta es una posibilidad especialmente interesante de evaluar a futuro en la medida que nos da elementos para pensar en la naturaleza de la relación entre estos dos grupos que compartieron un mismo territorio por tiempo considerable.

Agradecimientos: Este trabajo fue realizado como parte de las investigaciones del proyecto Fondecyt $\mathrm{N}^{\circ} 1090200$. Los análisis en el laboratorio de Arqueometría del Reactor de la Universidad de Missouri fueron financiados parcialmente por la subvención DBS-1110793 de la US National Science Foundation. Nuestros agradecimientos a Luis Cornejo por su ayuda con los test $\mathrm{z}$ y a dos evaluadores anónimos, cuyos comentarios fueron muy constructivos para mejorar este trabajo.

\section{Referencias Citadas}

Arnold, D.E. 1985. Ceramic Theory and Cultural Process. Cambridge University Press, Cambridge.

Cornejo, L., F. Falabella, L. Sanhueza e I. Correa 2012. Patrón de asentamiento durante el período Alfarero en la cuenca de Santiago, Chile Central. Una mirada a la escala local. Intersecciones en Antropología 13:449-60.

Falabella, F., L. Cornejo, I. Correa y L. Sanhueza 2014. Organización espacial durante el período Alfarero Temprano en Chile Central: un estudio a nivel de la localidad. En Distribución Espacial en Sociedades no Aldeanas: del Registro Arqueológico a la Interpretación Social, editado por F. Falabella, L. Sanhueza, L. Cornejo e I. Correa, pp. 51-88. Serie Monografías $\mathrm{N}^{\circ} 4$, Sociedad Chilena de Arqueología, Santiago.

Falabella, F., L. Cornejo, L. Sanhueza e I. Correa 2014. Trends in thermoluminescence date distributions for the Angostura micro region in Central Chile. Quaternary International, DOI: 10.1016/j.quaint.2014.06.049.

Falabella, F., I. Correa, L. Cornejo y L. Sanhueza 2012. Configuración de comunidades locales en los grupos del período alfarero temprano. Una propuesta metodológica y primeros resultados dentro de la cuenca del río Angostura. Actas XVIII Congreso Nacional de Arqueología Chilena, pp. 413-422. Sociedad Chilena de Arqueología, Santiago.

Falabella, F. y R. Stehberg 1989. Los inicios del desarrollo agrícola y alfarero: zona Central (300 a.C. a 900 d.C.). En Prehistoria, editado por J. Hidalgo, V. Schiappacasse, H. Niemeyer, C. Aldunate e I. Solimano, pp. 295-311. Editorial Andrés Bello, Santiago.

Falabella, F. y L. Sanhueza 2005-2006. Interpretaciones sobre la organización social de los grupos Alfareros Tempranos de 
Chile Central: alcances y perspectivas. Revista Chilena de Antropología 18:105-133.

Falabella, F., L. Sanhueza, I. Correa, M.D. Glascock, T.J. Ferguson y E. Fonseca 2013. Studying technological practices at a local level: neutron activation and petrographic analyses of Early Ceramic Period pottery in Central Chile. Archaeometry 55:33-53.

Glascock, M.D. 1992. Characterization of archaeological ceramics at MURR by Neutron Activation Analysis and multivariate statistics. En Chemical Characterization of Ceramic Pastes in Archaeology, editado por H. Neff, pp. 11-30. Prehistory Press, Madison.

Gosselain, O.P. 2000. Materializing identities: An African perspective. Journal of Archaeological Method and Theory 7:187-217

Gosselain, O.P. 2010. De l'art d'accomoder les pâtes et de s'accomoder d'autrui au sud du Niger. Espaces et échelles d'analyse. En Premiéres Sociétés Paysannes de la Méditerranée Orientale. Structure des Productions Céramiques, editado por C. Manen, F. Convertini, D. Bindere e I. Senepart, pp. 249-263. Mémoire 51 de la Société Préhistorique Francaise, Paris.

Johnson, A.W. y T.K. Earle 1987. The Evolution of Human Societies. From Foraging Group to Agrarian State. Stanford University Press, Stanford.

Lave, J. y E. Wenger 1991. Situated Learning: Legitimate Peripheral Participation, Cambridge University Press, New York.

Lechtman, H. 1977. Style in technology: some early thoughts. En Material Culture: Styles, Organization, and Dynamics of Technology, editado por H. Lechtman y R.S. Merrill, pp. 3-20. American Ethnological Society, St. Paul, Minnesota.

Lemonnier, P. 1986. The study of material culture today: Toward anthropology of technical systems. Journal of Anthropological Archaeology 5:147-186.

Lemonnier, P. 1992. Elements for an Anthropology of Technology. Museum of Anthropology, University of Michigan, Ann Arbor.

Neff, H. 2000. Neutron activation analysis for provenance determination in archaeology. En Modern Analytical Methods in Art and Archaeolog, editado por E. Ciliberto y G. Spoto, pp. 81-134. John Wiley \& Sons, New York.

Peterson, C.E. y R.D. Drennan 2005. Communities, settlements, sites, and surveys: Regional-scale analysis of prehistoric human interaction. American Antiquity 70:5-30.

Planella, M.T. y F. Falabella 1987. Nuevas perspectivas en torno al Período Alfarero Temprano en Chile Central. Clava 3:43-110.

Rice, P.M. 1987. Pottery Analysis: A Sourcebook. University of Chicago Press, Chicago.

Sanhueza, L. 2004. Estilos Tecnológicos e Identidades Sociales durante el Período Alfarero Temprano en Chile Central: Una Mirada desde la Alfarería. Tesis de Magister en Arqueología, Universidad de Chile, Santiago.

Sanhueza, L. 2013. Niveles de Integración Sociopolítica, Ideología e Interacción en Sociedades no Jerárquicas: Período Alfarero Temprano en Chile Central. Tesis de Doctorado, Universidad de Tarapacá-Universidad Católica del Norte, Arica-San Pedro de Atacama.

Sanhueza, L., L. Cornejo y F. Falabella 2007. Patrones de asentamiento en el período Alfarero Temprano de Chile Central. Chungara Revista de Antropología Chilena 39:103-115.

Sanhueza, L. y F. Falabella 2007. Hacia una inferencia de las relaciones sociales del Complejo Llolleo durante el período Afarero Temprano en Chile Central. En Procesos Sociales Prehispánicos en el Sur Andino: la Vivienda, la Comunidad y el Territorio, editado por A. Nielsen, C. Rivolta, V. Seldes, M. Vásquez y P. Mercolli, pp. 377-92. Editorial Brujas, Córdoba.

Sanhueza, L., M. Vásquez y F. Falabella 2003. Las sociedades alfareras tempranas de la cuenca de Santiago. Chungara Revista de Antropología Chilena 35:23-50.

Sellés, D. y P. Gana 2001. Geología del Área Talagante-San Francisco de Mostazal. Carta Geológica de Chile, Serie Geología Básica, No 74. SERNAGEOMIN, Santiago.

Shepard, A.O. 1980 [1954]. Ceramics for the Archaeologist. Carnegie Institution of Washington, Washington D.C.

Stark, M.T. (ed.) 1998. The Archaeology of Social Boundaries. Smithsonian Institution Press, Washington D.C.

\section{Nota}

1 Los primeros resultados de este estudio por activación neutrónica, referidos solo a la localidad de Valdivia de Paine, fueron publicados en Falabella et al. 2013. 